\title{
Exact Traveling Wave Solutions of
} Nano-Ionic Solitons and Nano-Ionic Current of MTs Using the $\exp (-\varphi(\xi))$-Expansion Method

\author{
Emad H. M. Zahran \\ Department of Mathematical and Physical Engineering, College of Engineering, Shubra University of Benha, \\ Egypt \\ Email: e__h_zahran@hotmai1.com
}

Received 3 January 2015; accepted 16 April 2015; published 22 April 2015

Copyright (C) 2015 by author and Scientific Research Publishing Inc.

This work is licensed under the Creative Commons Attribution International License (CC BY).

http://creativecommons.org/licenses/by/4.0/

(c) (i) Open Access

\section{Abstract}

In this work, the $\exp (-\varphi(\xi))$-expansion method is used for the first time to investigate the exact traveling wave solutions involving parameters of nonlinear evolution equations. When these parameters are taken to be special values, the solitary wave solutions are derived from the exact traveling wave solutions. The validity and reliability of the method are tested by its applications to Nano-ionic solitons wave's propagation along microtubules in living cells and Nano-ionic currents of MTs which play an important role in biology.

\section{Keywords}

The $\exp (-\varphi(\xi))$-Expansion Method, Nano-Solitons of Ionic Wave's Propagation along

Microtubules in Living Cells, Nano-Ionic Currents of MTs, Traveling Wave Solutions, Kink and Anti Kink Wave Solutions

\section{Introduction}

The nonlinear partial differential equations of mathematical physics are major subjects in physical science [1]. Exact solutions for these equations play an important role in many phenomena in physics such as fluid mechanics, hydrodynamics, Optics, Plasma physics and so on. Recently many new approaches for finding these soluHow to cite this paper: Zahran, E.H.M. (2015) Exact Traveling Wave Solutions of Nano-lonic Solitons and Nano-lonic Current of MTs Using the exp $(-\varphi(\xi))$-Expansion Method. Advances in Nanoparticles, 4, 25-36. 
tions have been proposed, for example, tanh-sech method [2]-[4], extended tanh-method [5]-[7], sine-cosine method [8]-[10], homogeneous balance method [11] [12], F-expansion method [13]-[15], exp-function method [16] [17], trigonometric function series method [18], $\left(\frac{G^{\prime}}{G}\right)$ expansion method [19]-[22], Jacobi elliptic function method [23]-[26], The $\exp (-\varphi(\xi))$-expansion method [27]-[29] and so on.

The objective of this article is to investigate more applications than obtained in [27]-[29] to justify and demonstrate the advantages of the $\exp (-\varphi(\xi))$-method. Here, we apply this method to Nano-solitons of ionic waves's propagation along microtubules in living cells and Nano-ionic currents of MTs.

\section{Description of Method}

Consider the following nonlinear evolution equation

$$
f\left(u, u_{t}, u_{x}, u_{t t}, u_{x x}, \cdots\right)=0,
$$

where $F$ is a polynomial in $u(x, t)$ and its partial derivatives in which the highest order derivatives and nonlinear terms are involved. In the following, we give the main steps of this method.

Step 1. We use the wave transformation

$$
u(x, t)=u(\xi), \xi=x-c t,
$$

where $c$ is a positive constant, to reduce Equation (2.1) to the following ODE:

$$
p\left(u, u^{\prime}, u^{\prime \prime}, u^{\prime \prime \prime}, \cdots\right)=0 \text {, }
$$

where $P$ is a polynomial in $u(\xi)$ and its total derivatives.

Step 2. Suppose that the solution of ODE (2.3) can be expressed by a polynomial in $\exp (-\varphi(\xi))$ as follow

$$
u(\xi)=a_{m}(\exp (-\varphi(\xi)))^{m}+\cdots, a_{m} \neq 0,
$$

where $\varphi(\xi)$ satisfies the ODE in the form

$$
\varphi^{\prime}(\xi)=\exp (-\varphi(\xi))+\mu \exp (\varphi(\xi))+\lambda,
$$

The solutions of ODE (2.5) are

When $\lambda^{2}-4 \mu>0, \mu \neq 0$,

$$
\varphi(\xi)=\ln \left(\frac{-\sqrt{\lambda^{2}-4 \mu} \tanh \left(\frac{\sqrt{\lambda^{2}-4 \mu}}{2}\left(\xi+c_{1}\right)\right)-\lambda}{2 \mu}\right),
$$

When $\lambda^{2}-4 \mu>0, \mu=0$,

$$
\varphi(\xi)=-\ln \left(\frac{\lambda}{\exp \left(\lambda\left(\xi+C_{1}\right)\right)-1}\right),
$$

When $\lambda^{2}-4 \mu=0, \mu \neq 0, \lambda \neq 0$,

$$
\varphi(\xi)=\ln \left(-\frac{2\left(\lambda\left(\xi+C_{1}\right)+2\right)}{\lambda^{2}\left(\xi+C_{1}\right)}\right),
$$

When $\lambda^{2}-4 \mu=0, \mu \neq 0, \lambda=0$,

$$
\varphi(\xi)=\ln \left(\xi+C_{1}\right)
$$

When $\lambda^{2}-4 \mu<0$ 


$$
\varphi(\xi)=\ln \left(\frac{\sqrt{4 \mu-\lambda^{2}} \tan \left(\frac{\sqrt{4 \mu-\lambda^{2}}}{2}\left(\xi+c_{1}\right)\right)-\lambda}{2 \mu}\right),
$$

where $a_{m}, \cdots, \lambda, \mu$ are constants to be determined later,

Step 3. Substitute Equation (2.4) along Equation (2.5) into Equation (2.3) and collecting all the terms of the same power $\exp (-m \varphi(\xi)), \quad(m=0,1,2,3, \cdots)$ and equating them to zero, we obtain a system of algebraic equations, which can be solved by Maple or Mathematica to get the values of.

Step 4. substituting these values and the solutions of Equation (2.5) into Equation (2.3) we obtain the exact solutions of Equation (2.1).

\section{Application}

\subsection{Example 1: Nano-Solitons of Ionic Wave's Propagation along Microtubules in Living Cells [27]}

We first consider an inviscid, incompressible and non-rotating flow of fluid of constant depth $(h)$. We take the direction of flow as $x$-axis and $z$-axis positively upward the free surface in gravitational field. The free surface elevation above the undisturbed depth $h$ is $n(x, t)$, so that the wave surface at height $z=h+\eta(x, t)$, while $z=$ 0 is horizontal rigid bottom.

Let $\phi(x, z, t)$ be the scalar velocity potential of the fluid lying between the bottom $(z=0)$ and free space $\eta(x, t)$, then we could write the Laplace and Euler equation with the boundary conditions at the surface and the bottom, respectively, as follows:

$$
\begin{gathered}
\frac{\partial^{2} \varphi}{\partial x^{2}}+\frac{\partial^{2} \varphi}{\partial z^{2}}=0 ; 0 \prec z \prec h+\eta ;-\infty \prec x \prec+\infty \\
\frac{\partial \varphi}{\partial t}+\frac{1}{2}\left(\frac{\partial \varphi}{\partial z} \boldsymbol{i}+\frac{\partial \varphi}{\partial z} \boldsymbol{k}\right)^{2}+g \eta=0 ; \quad z=h+\eta \\
\frac{\partial \eta}{\partial t}+\frac{\partial \eta}{\partial x} \frac{\partial \varphi}{\partial x}-\frac{\partial \varphi}{\partial z}=0, \\
\frac{\partial \varphi}{\partial z}=0 ; \quad z=0 .
\end{gathered}
$$

It is useful to introduce two following fundamental dimensionaless parameters:

$$
\sigma=\frac{\eta_{0}}{h} \prec 1 ; \delta=\left(\frac{h}{l}\right)^{2} \prec 1,
$$

where $\eta_{0}$ is the wave amplitude, and $l$ is the characteristic length-like wavelength. Accordingly, we also take a complete set of new suitable non-dimensional variables:

$$
\chi=\frac{x}{l} ; Z=\frac{z}{h} ; T=\frac{c t}{l} ; \psi=\frac{\eta}{\eta_{0}} ; \phi=\frac{h}{\eta_{0} l_{c}} \varphi,
$$

where $c=\sqrt{g h}$ is the shallow-water wave speed, with g being gravitational acceleration. In term of (3.5) and (3.6) the initial system of Equations (3.1)-(3.4) now reads

$$
\begin{gathered}
\delta \frac{\partial^{2} \phi}{\partial x^{2}}+\frac{\partial^{2} \phi}{\partial z^{2}}=0 \\
\frac{\partial \phi}{\partial \tau}+\frac{\sigma}{2}\left(\frac{\partial \phi}{\partial x}\right)^{2}+\frac{\sigma}{2 \delta}\left(\frac{\partial \phi}{\partial x}\right)^{2}+\psi=0 ; Z=1+\sigma \psi,
\end{gathered}
$$




$$
\begin{gathered}
\frac{\partial \psi}{\partial \tau}+\sigma\left(\frac{\partial \phi}{\partial \chi} \frac{\partial \psi}{\partial \chi}\right)-\frac{1}{\delta}\left(\frac{\partial \phi}{\partial Z}\right)=0 ; Z=1+\sigma \psi, \\
\frac{\partial \phi}{\partial z}=0 ; \quad z=0 .
\end{gathered}
$$

Expanding $\phi(x, t)$ in terms of $\delta$

$$
\phi=\phi_{0}+\delta \phi_{1}+\delta^{2} \phi_{2}
$$

and using the dimensionless wave particles velocity in $x$-direction, by definition $u=\frac{\partial_{\phi}}{\partial \chi}$, then substituting of (3.11) into (3.7)-(3.9), with retaining terms up to linear order of small parameters $(\delta, \sigma)$ in (3.8), and second order in (3.9), we get

$$
\begin{gathered}
\frac{\partial \phi_{0}}{\partial \tau}+\frac{\delta}{2}\left(\frac{\partial^{2} u}{\partial_{\tau} \partial x}\right)+\psi+\frac{1}{2} \sigma u^{2}=0 \\
\frac{\partial \psi}{\partial \tau}+\sigma u\left(\frac{\partial \psi}{\partial x}\right)+\frac{1}{\delta}(1+\sigma \psi) \frac{\partial u}{\partial x}=\frac{\delta}{6} \frac{\partial^{3} u}{\partial x^{3}} .
\end{gathered}
$$

Making the differentiation of (3.12) with respect to $\chi$, and rearranging (3.13), we get

$$
\begin{gathered}
\frac{\partial u}{\partial \tau}+\sigma u\left(\frac{\partial u}{\partial x}\right)+\frac{\partial \psi}{\partial x}-\frac{1}{2} \delta \frac{\partial^{3} u}{\partial x^{2} \partial \tau}=0, \\
\frac{\partial \psi}{\partial \tau}+\frac{\partial}{\partial \chi}[u(1+\sigma \psi)]-\frac{1}{6} \delta \frac{\partial^{3} u}{\partial x^{3}}=0,
\end{gathered}
$$

Returning back to dimensional variables $\eta(x, t)$ and $v=\frac{\mathrm{d} \phi}{\mathrm{d} x}$, (3.14) now reads

$$
\frac{\partial v}{\partial \tau}+v\left(\frac{\partial v}{\partial x}\right)+g \frac{\partial \eta}{\partial x}=\frac{1}{3} h^{2} \frac{\partial^{3} v}{\partial x^{2} \partial t}
$$

We could define the new function $V(x, t)$ unifying the velocity and displacement of water particles as follows:

$$
v=\frac{1}{h}\left(\frac{\partial V}{\partial t}\right) ; \eta=\frac{\partial V}{\partial x}
$$

implying that (3.16) becomes

$$
\frac{\partial^{2} V}{\partial t^{2}}-g h \frac{\partial^{2} V}{\partial x^{2}}+\frac{1}{2 h} \frac{\partial}{\partial x}\left(\frac{\partial V}{\partial t}\right)^{2}=\frac{1}{3} h^{2} \frac{\partial^{4} V}{\partial x^{2} \partial t^{2}}
$$

We seek for traveling wave solutions with moving coordinate of the form $\xi=x-v t$ and with wave speed $v$, which reduces Equation (3.18) into ordinary nonlinear differential equation as follows:

$$
\left(v^{2}-g h\right) \frac{\partial^{2} V}{\partial \xi^{2}}+\frac{v^{2}}{2 h} \frac{\partial}{\partial \xi}\left(\frac{\partial V}{\partial \xi}\right)^{2}=\frac{1}{3} h^{2} v^{2} \frac{\partial^{4} V}{\partial \xi^{4}}
$$

Integrating Equation (3.19) once, and setting $\frac{\partial V}{\partial \xi}=W$, we get

$$
\frac{\partial^{2} W}{\partial \xi^{2}}=\alpha W^{2}+\beta W+c_{1} .
$$

Balancing $W^{\prime \prime}$ and $W^{2}$ yields, $N+2=2 N \Rightarrow N=2$. Therefore, we can write the solution of Equation 
(3.20) in the form

$$
\begin{gathered}
W(\xi)=a_{0}+a_{1} \mathrm{e}^{-\phi(\xi)}+a_{2} \mathrm{e}^{-2 \phi(\xi)}, \\
W^{\prime}(\xi)=-\frac{a_{1}}{\left(\mathrm{e}^{\phi(\xi)}\right)^{2}}-a_{1} \mu-\frac{a_{1} \lambda}{\mathrm{e}^{\phi(\xi)}}-2 \frac{a_{2}}{\left(\mathrm{e}^{\phi(\xi)}\right)^{3}}-2 \frac{a_{2} \mu}{\mathrm{e}^{\phi(\xi)}}-2 \frac{a_{2} \lambda}{\left(\mathrm{e}^{\phi(\xi)}\right)^{2}}, \\
W^{\prime \prime}(\xi)=2 \frac{a_{1}}{\left(\mathrm{e}^{\phi(\xi)}\right)^{3}}+2 \frac{a_{1} \mu}{\mathrm{e}^{\phi(\xi)}}+3 \frac{a_{1} \lambda}{\left(\mathrm{e}^{\phi(\xi)}\right)^{2}}+a_{1} \lambda \mu+\frac{a_{1} \lambda^{2}}{\mathrm{e}^{\phi(\xi)}}+6 \frac{a_{2}}{\left(\mathrm{e}^{\phi(\xi)}\right)^{4}}+8 \frac{a_{2 \mu}}{\left(\mathrm{e}^{\phi(\xi)}\right)^{2}} \\
+10 \frac{a_{2} \lambda}{\left(\mathrm{e}^{\phi(\xi)}\right)^{3}}+2 a_{2} \mu^{2}+6 \frac{a_{2} \mu \lambda}{\mathrm{e}^{\phi(\xi)}}+4 \frac{a_{2} \lambda^{2}}{\left(\mathrm{e}^{\phi(\xi)}\right)^{2}}
\end{gathered}
$$

Substituting (3.21) along (3.23) into (3.20), setting the coefficients of $\exp (-4 \varphi(C \xi)), \exp (-3 \varphi(\xi))$, $\exp (-2 \varphi(\xi)), \exp (-1 \varphi(\xi))$, $\exp (0 \varphi(\xi))$ to zero, we obtain the following underdetermined system of algebraic equations for $\left(a_{0}, a_{1}, a_{2}\right)$ :

$$
\begin{gathered}
6 a_{2}-\alpha a_{2}^{2}=0, \\
2 a_{1}+10 a_{2} \lambda-2 \alpha a_{1} a_{2}=0, \\
3 a_{1} \lambda+8 a_{2} \mu+4 a_{2} \lambda^{2}-2 \alpha a_{0} a_{2}-\alpha a_{1}^{2}-\beta a_{2}=0, \\
2 a_{1} \mu+a_{1} \lambda^{2}+6 a_{2} \mu \lambda-2 \alpha a_{0} a_{1}-\beta a_{1}=0, \\
a_{1} \lambda \mu+2 a_{2} \mu^{2}-\alpha a_{2}^{2}-\beta a_{0}-c_{1}=0,
\end{gathered}
$$

Solving the above system with the aid of Mathematica or Maple, we have the following solution:

$$
\begin{gathered}
c_{1}=\frac{-3 a_{1}^{4}+24 a_{1}^{2} a_{0} a_{2}+2 a_{1}^{2} \beta a_{2}^{2}+144 a_{0}^{2} a_{2}^{2}-8 \beta a_{0} a_{2}^{3}+\beta^{2} a_{2}^{4}-192 a_{2}^{4}}{32 a_{2}^{3}}, \\
\mu=\frac{-a_{1}^{2}+12 a_{0} a_{2}+\beta a_{2}^{2}}{8 a_{2}}, a_{0}=a_{0}, a_{1}=\frac{6}{\alpha}, a_{2}=\frac{6 \lambda}{\alpha} .
\end{gathered}
$$

Sothat the solution of Equation (3.20) will be in the form:

$$
W(\xi)=a_{0}+\frac{6}{\alpha} \exp (-\varphi(\xi))+\frac{6 \lambda}{\alpha} \exp (-2 \varphi(\xi)),
$$

Consequently, the solution takes the forms:

When $\lambda^{2}-4 \mu>0, \mu \neq 0$,

$$
u=a_{0}+\frac{6}{\alpha} \frac{2 \mu}{-\sqrt{\lambda^{2}-4 \mu} \tanh \left(\frac{\sqrt{\lambda^{2}-4 \mu}}{2}\left(\xi+C_{1}\right)\right)-\lambda}+\frac{6 \lambda}{\alpha}\left(2 \mu-\sqrt{\lambda^{2}-4 \mu} \tanh \left(\frac{\sqrt{\lambda^{2}-4 \mu}}{2}\left(\xi+C_{1}\right)\right)-\lambda\right)^{2}
$$

When $\lambda^{2}-4 \mu>0, \mu=0$,

$$
u=a_{0}+\frac{6}{\alpha} \frac{\lambda}{\exp \left(\lambda\left(\xi+C_{1}\right)\right)-1}+\frac{6 \lambda}{\alpha}\left(\frac{\lambda}{\exp \left(\lambda\left(\xi+C_{1}\right)\right)-1}\right)^{2},
$$

When $\lambda^{2}-4 \mu=0, \mu \neq 0, \lambda \neq 0$,

$$
u=a_{0}-\frac{6}{\alpha} \frac{2\left(\lambda\left(\xi+C_{1}\right)+2\right)}{\lambda^{2}\left(\xi+C_{1}\right)}+\frac{6 \lambda}{\alpha}\left(\frac{2\left(\lambda\left(\xi+C_{1}\right)+2\right)}{\lambda^{2}\left(\xi+C_{1}\right)}\right)^{2},
$$


When $\lambda^{2}-4 \mu=0, \mu=0, \lambda=0$,

$$
u=a_{0}+\frac{6}{\alpha} \frac{1}{\xi+C_{1}}+\frac{6 \lambda}{\alpha}\left(\frac{1}{\xi+C_{1}}\right)^{2}
$$

When $\lambda^{2}-4 \mu<0$,

$$
\begin{gathered}
u=a_{0}+\frac{6}{\alpha} \frac{2 \mu}{\sqrt{4 \mu-\lambda^{2}} \tan \left(\frac{\sqrt{4 \mu-\lambda^{2}}}{2}\left(\xi+C_{1}\right)\right)-\lambda} \\
+\frac{6 \lambda}{\alpha}\left(\frac{2 \mu}{\sqrt{4 \mu-\lambda^{2}} \tan \left(\frac{\sqrt{4 \mu-\lambda^{2}}}{2}\left(\xi+C_{1}\right)\right)-\lambda}\right)^{2},
\end{gathered}
$$

\subsection{Example 2. Nano-Ionic Currents of MTs}

The Nano-ionic currents are elaborated in [27] take the form

$$
\frac{l^{2}}{3} u_{x x x}+\frac{\mathcal{Z}^{\frac{3}{2}}}{l}\left(x c_{0}-2 s s_{0}\right) u u_{t}+2 u+\frac{z c_{0}}{l} u_{t}+\frac{1}{l}\left(R z^{-1}-G_{0} z\right) u=0,
$$

where $R=0.34 \times 10^{9} \Omega$ is the resistance of the ER with length, $l=8 \times 19^{-9} \mathrm{~m}, c_{0}=1.8 \times 10^{-15} \mathrm{~F}$ is the maximal capacitance of the ER, $G_{0}=1.1 \times 10^{-13}$ si is conductance of pertaining NPs and $z=5.56 \times 10^{10} \Omega$ is the characteristic impedance of our system parameters $\delta$ and $\chi$ describe nonlinearity of ER capacitor and conductance of NPs in ER, respectively. In order to solve Equation (3.35) we use the travelling wave transformations $u(x, t)=u(\xi), \quad \xi=\frac{1}{l} x-\frac{C}{\tau} t$, with $\tau=R C_{0}=0.6 \times 10^{-6} \mathrm{~s}$, to reduce Equation (3.35) to the following nonlinear ordinary differential equation:

$$
\frac{1}{3} u^{\prime \prime \prime}-\frac{c z^{\frac{3}{2}}}{\tau}\left(x c_{0}-2 s c_{0}\right) u u^{\prime}+\left(2-\frac{c_{0} c z}{\tau}\right) u^{\prime}+\left(\frac{R}{z}-G_{0} z\right) u=0,
$$

Which can be written in the form

$$
\frac{1}{3} u^{\prime \prime \prime}+H_{1} u u^{\prime}+H_{2} u^{\prime}+H_{3} u=0
$$

where

$$
H_{1}=\frac{c}{\tau} B, H_{2}=\left(2-\frac{c e}{\tau}\right), B=-z^{\frac{3}{2}}\left(x c_{0}-2 s s_{0}\right), E=c_{0} z, D=H_{3},
$$

Thus Equation (3.37) take the form

$$
\frac{1}{3} u^{\prime \prime \prime}+\frac{c}{\tau} B u u^{\prime}+\left(2-\frac{c E}{\tau}\right) u^{\prime}+D u=0,
$$

Balancing $u^{\prime \prime \prime}$ and $u u^{\prime}$ yields, $N+3=N+N+1 \rightarrow N=2$. Consequently, we get

$$
u=a_{0}+a_{1} \mathrm{e}^{-\phi(\xi)}+a_{2} \mathrm{e}^{-2 \phi(\xi)},
$$

Where $a_{0}, a_{1}, a_{2}$ are arbitrary constants such that $a_{2} \neq 0$. From Equation (3.40), it is easy to see that 


$$
\begin{aligned}
u^{\prime}= & -\frac{a_{1}}{\left(\mathrm{e}^{\phi(\xi)}\right)^{2}}-a_{1} \mu-\frac{a_{1} \lambda}{\mathrm{e}^{\phi(\xi)}}-2 \frac{a_{2}}{\left(\mathrm{e}^{\phi(\xi)}\right)^{3}}-2 \frac{a_{2} \mu}{\mathrm{e}^{\phi(\xi)}}-2 \frac{a_{2} \lambda}{\left(\mathrm{e}^{\phi(\xi)}\right)^{2}}, \\
W^{\prime \prime}(\xi)= & 2 \frac{a_{1}}{\left(\mathrm{e}^{\phi(\xi)}\right)^{3}}+2 \frac{a_{1} \mu}{\mathrm{e}^{\phi(\xi)}}+3 \frac{a_{1} \lambda}{\left(\mathrm{e}^{\phi(\xi)}\right)^{2}}+a_{1} \lambda \mu+\frac{a_{1} \lambda^{2}}{\mathrm{e}^{\phi(\xi)}}+6 \frac{a_{2}}{\left(\mathrm{e}^{\phi(\xi)}\right)^{4}} \\
& +8 \frac{a_{2} \mu}{\left(\mathrm{e}^{\phi(\xi)}\right)^{2}}+10 \frac{a_{2} \lambda}{\left(\mathrm{e}^{\phi(\xi)}\right)^{3}}+2 a_{2} \mu^{2}+6 \frac{a_{2 \mu} \lambda}{\mathrm{e}^{\phi(\xi)}}+4 \frac{a_{2} \lambda^{2}}{\left(\mathrm{e}^{\phi(\xi)}\right)^{2}},
\end{aligned}
$$

Substituting Equations (3.40)-(3.42) into Equation (3.39) and equating the coefficients of $\exp (-5 \varphi(\xi))$, $\exp (-4 \varphi(\xi)), \exp (-3 \varphi(\xi)), \exp (-2 \varphi(\xi)), \exp (-1 \varphi(\xi)), \exp (-0 \varphi(\xi))$ to zero, we obtain

$$
\begin{gathered}
-8 a_{2}-2 \frac{c B a_{2}^{2}}{\tau}=0 \\
-2 a_{1}-18 a_{2} \lambda+\frac{c B\left(-3 a_{1} a_{2}-2 a_{2}^{2} \lambda\right)}{\tau}=0 \\
-\frac{38}{3} a_{2} \lambda^{2}-4 a_{1} \lambda-\frac{40}{3} a_{2} \mu \\
+\frac{c B\left(-2 a_{0} a_{2}-a_{1}^{2}-3 a_{0} a_{2} \lambda-2 a_{2}^{2} \mu\right)}{\tau}-2\left(2-\frac{c E}{\tau}\right) a_{2}=0, \\
-\frac{8}{3} a_{1} \mu-\frac{8}{3} a_{2} \lambda^{3}-\frac{7}{3} a_{1} \lambda^{2}-\frac{52}{3} a_{2} \mu \lambda \\
+\frac{c B\left(-a_{0} a_{1}-2 a_{0} a_{2} \lambda-a_{1}^{2} \lambda-3 a_{1} a_{2} \mu\right)}{\tau} \\
+\left(2-\frac{c E}{\tau}\right)\left(-a_{1}-2 a_{2} \lambda\right)+(D) a_{2}=0 \\
-\frac{1}{3} a_{1} \lambda^{3}-\frac{14}{3} a_{2} \mu \lambda^{2}-\frac{8}{3} a_{1} \lambda \mu-\frac{16}{3} a_{2} \mu^{2} \\
+\frac{c B\left(-a_{0} a_{1} \lambda-2 a_{0} a_{2} \mu-a_{1}^{2} \mu\right)}{\tau} \\
+\left(2-\frac{c E}{\tau}\right)\left(-a_{1} \lambda-2 a_{2} \mu\right)+(D) a_{1}=0 \\
-\frac{2}{3} a_{1} \mu^{2}-2 a_{2} \mu^{2} \lambda-\frac{1}{3} a_{1} \lambda^{2} \mu-\frac{c B a_{0} a_{1} \mu}{\tau}-\left(2-\frac{c E}{\tau}\right) a_{1} \mu+(D) a_{0}=0
\end{gathered}
$$

Solving above system with the aid of Mathematica or Maple, we have the following solution:

$$
\begin{aligned}
& B=-4 \frac{\tau}{c a_{2}}, D=0, \lambda=\frac{a_{1}}{a_{2}}, \\
& a_{0}=-\frac{1}{12} \frac{-\tau a_{1}^{2}-8 a_{2}^{2} \mu \tau-6 a_{2}^{2} \tau+3 a_{2}^{2} c E}{a_{2} \tau},
\end{aligned}
$$

$a_{1}=a_{1}, a_{2}=a_{2}$.

So that the solution of Equation (3.39) will be in the form: 


$$
u=-\frac{1}{12} \frac{-\tau a_{1}^{2}-8 a_{2}^{2} \mu \tau-6 a_{2}^{2} \tau+3 a_{2}^{2} c E}{a_{2} \tau}+a_{1} \exp (-\varphi(\xi))+a_{2} \exp (-2 \varphi(\xi)),
$$

Consequently, the solution take the forms:

When $\lambda^{2}-4 \mu>0, \mu \neq 0$,

$$
\begin{aligned}
u= & -\frac{1}{12} \frac{-\tau a_{1}^{2}-8 a_{2}^{2} \mu \tau-6 a_{2}^{2} \tau+3 a_{2}^{2} c E}{a_{2} \tau}+a_{1} \frac{2 \mu}{-\sqrt{\lambda^{2}-4 \mu} \tanh \left(\frac{\sqrt{\lambda^{2}-4 \mu}}{2}\left(\xi+C_{1}\right)\right)-\lambda} \\
& +a_{2}\left(\frac{2 \mu}{-\sqrt{\lambda^{2}-4 \mu} \tanh \left(\frac{\sqrt{\lambda^{2}-4 \mu}}{2}\left(\xi+C_{1}\right)\right)-\lambda}\right)^{2}
\end{aligned}
$$

When $\lambda^{2}-4 \mu>0, \mu=0$,

$$
u=-\frac{1}{12} \frac{-\tau a_{1}^{2}-8 a_{2}^{2} \mu \tau-6 a_{2}^{2} \tau+3 a_{2}^{2} c E}{a_{2} \tau}+a_{1} \frac{\lambda}{\exp \left(\lambda\left(\xi+C_{1}\right)\right)-1}+a_{2}\left(\frac{\lambda}{\exp \left(\lambda\left(\xi+C_{1}\right)\right)-1}\right)^{2},
$$

When $\lambda^{2}-4 \mu>0, \mu \neq 0, \lambda \neq 0$,

$$
u=-\frac{1}{12} \frac{-\tau a_{1}^{2}-8 a_{2}^{2} \mu \tau-6 a_{2}^{2} \tau+3 a_{2}^{2} c E}{a_{2} \tau}-a_{1} \frac{2\left(\lambda\left(\xi+C_{1}\right)+2\right)}{\lambda^{2}\left(\xi+C_{1}\right)}+a_{2}\left(\frac{2\left(\lambda\left(\xi+C_{1}\right)+2\right)}{\lambda^{2}\left(\xi+C_{1}\right)}\right)^{2},
$$

When $\lambda^{2}-4 \mu=0, \mu=0, \lambda=0$,

$$
u=-\frac{1}{12} \frac{-\tau a_{1}^{2}-8 a_{2}^{2} \mu \tau-6 a_{2}^{2} \tau+3 a_{2}^{2} c E}{a_{2} \tau}+a_{1} \frac{1}{\xi+C_{1}}+a_{2}\left(\frac{1}{\xi+C_{1}}+a_{2}\right)^{2},
$$

When $\lambda^{2}-4 \mu<0$,

$$
\begin{aligned}
u= & -\frac{1}{12} \frac{-\tau a_{1}^{2}-8 a_{2}^{2} \mu \tau-6 a_{2}^{2} \tau+3 a_{2}^{2} c E}{a_{2} \tau}+a_{1} \frac{2 \mu}{\sqrt{4 \mu-\lambda^{2}} \tan \left(\frac{\sqrt{4 \mu-\lambda^{2}}}{2}\left(\xi+C_{1}\right)\right)-\lambda} \\
& +a_{2}\left(\frac{2 \mu}{\sqrt{4 \mu-\lambda^{2}} \tan \left(\frac{\sqrt{4 \mu-\lambda^{2}}}{2}\left(\xi+C_{1}\right)\right)-\lambda}\right)^{2}
\end{aligned}
$$

\section{Results and Conclusion}

In nanobiosciences the transmission line models for ionic waves propagating along microtubules in living cells play an important role in cellular signaling where ionic wave's propagating along microtubules in living cells shaped as nanotubes that are essential for cell motility, cell division, intracellular trafficking and information processing within neuronal processes. ionic waves propagating along microtubules in living cells have been also implicated in higher neuronal functions, including memory and the emergence of consciousness and we presented an inviscid, incompressible and non-rotating flow of fluid of constant depth $(h)$. The $\exp (-\varphi(\xi))$-expansion method has been successfully used to find the exact traveling wave solutions of some nonlinear evolution equations and Figure 1 and Figure 2 show the solitary wave solution of both equations. As an application, 


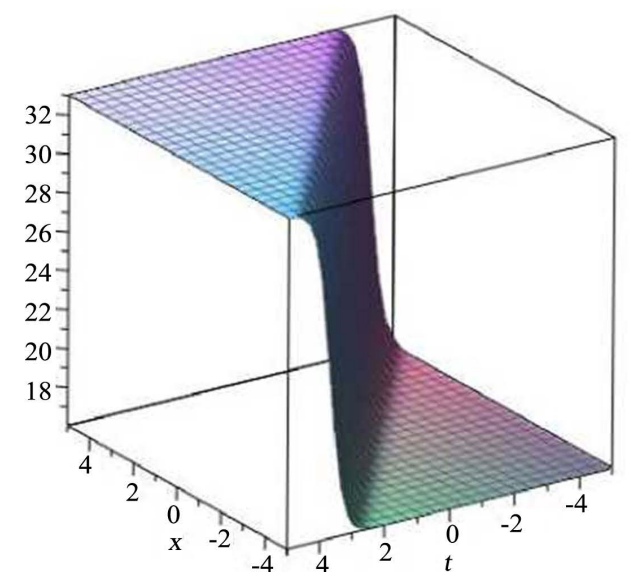

(a)

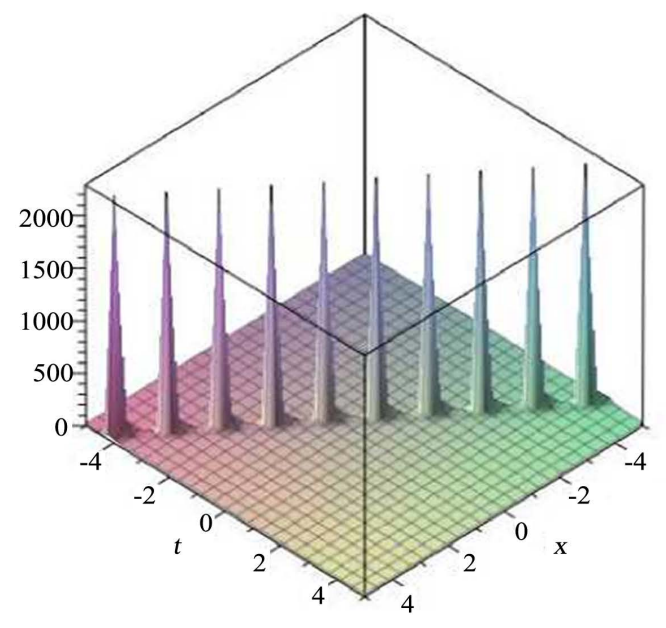

(c)

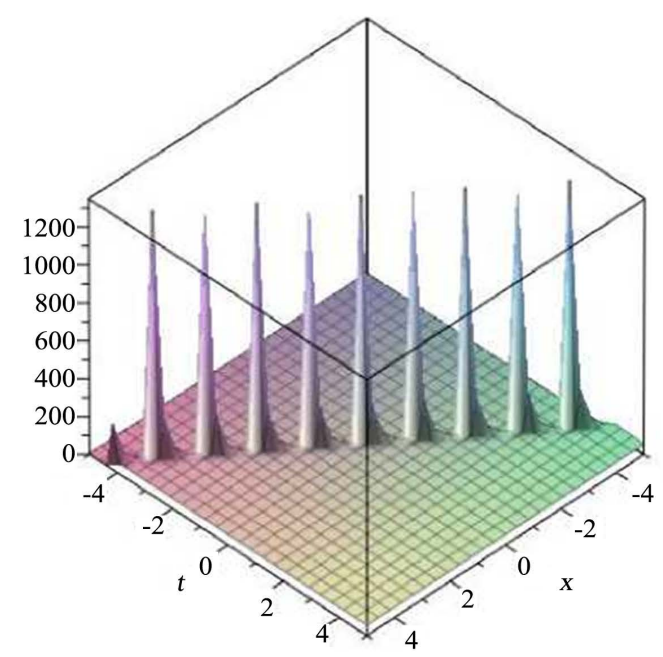

(b)

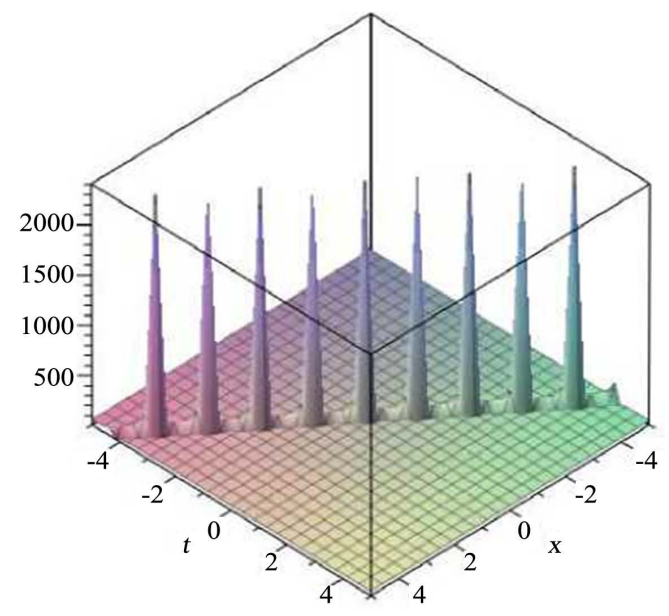

(d)

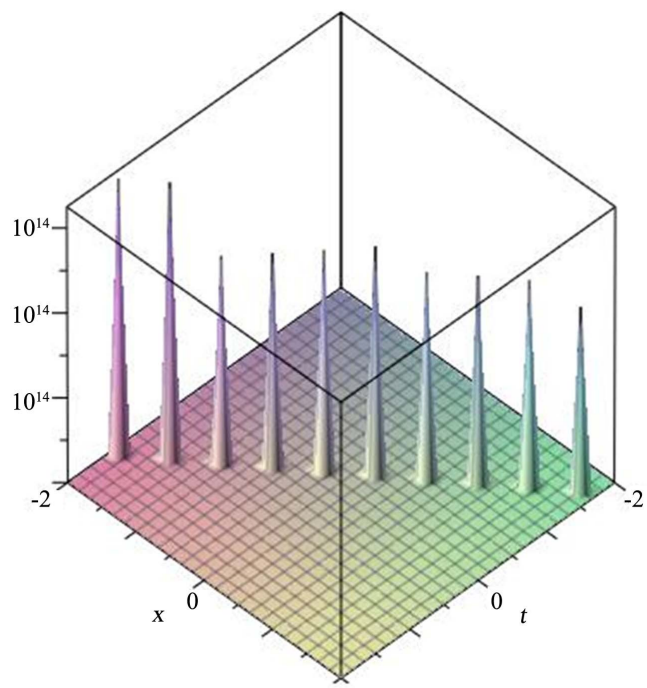

(e)

Figure 1. Solution of Equations (3.30)-(3.34). (a) Equation (3.30); (b) Equation (3.31); (c) Equation (3.32); (d) Equation (3.33); (e) Equation (3.34). 


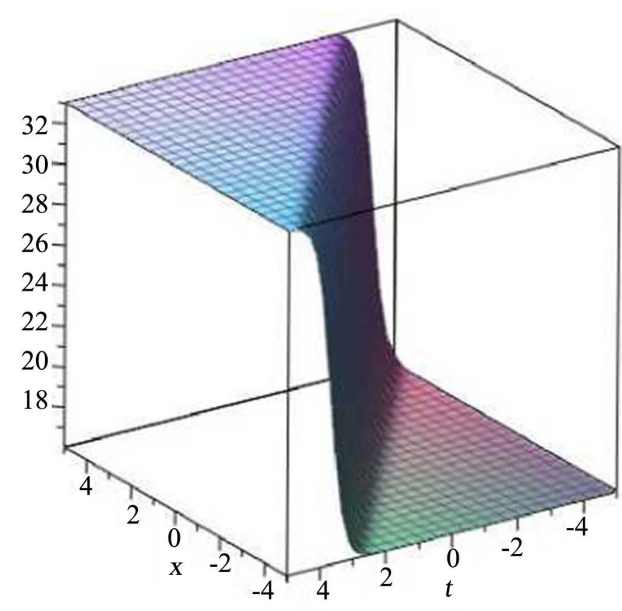

(a)

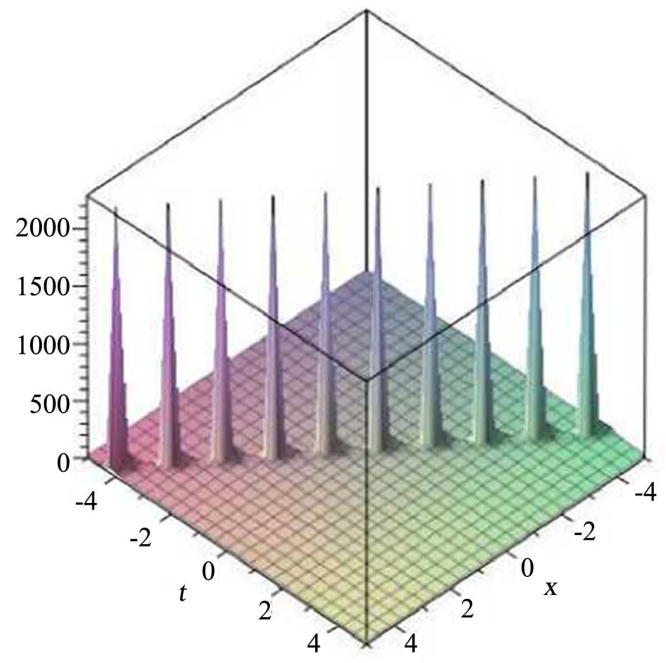

(c)

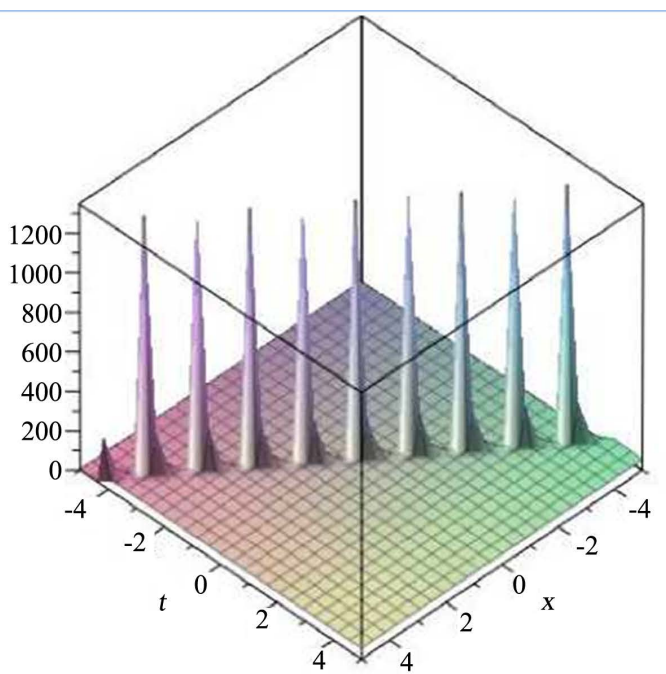

(b)

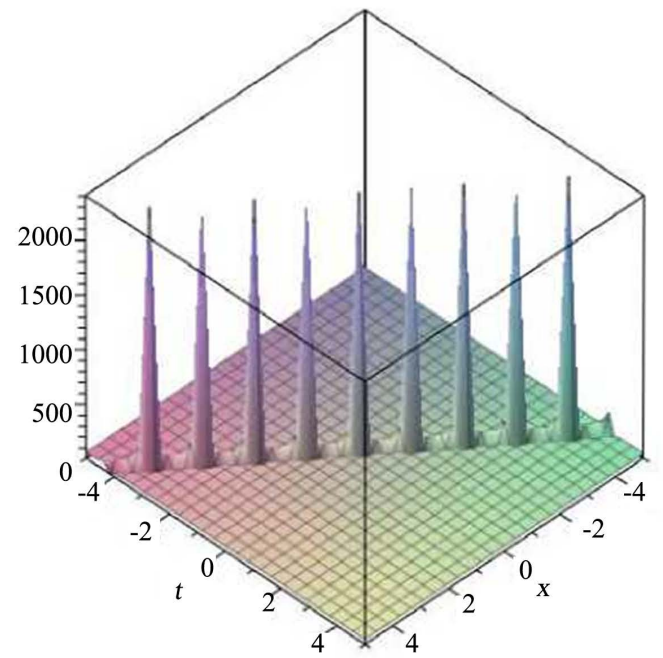

(d)

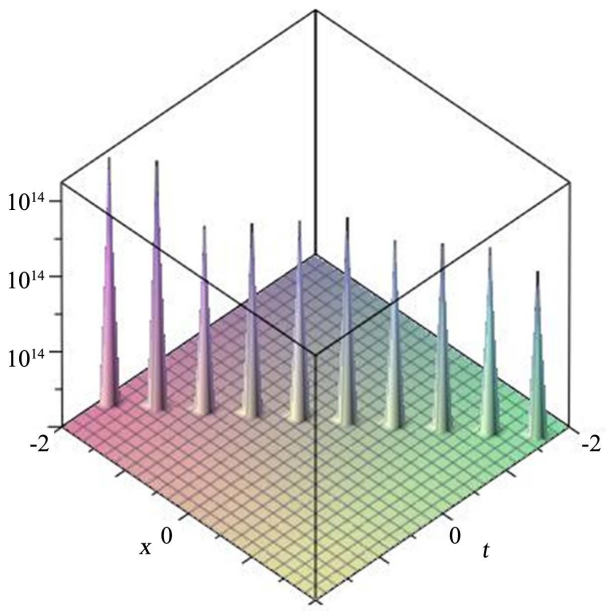

(e)

Figure 2. Solutiou of Equations (3.50)-(3.54). (a) Equation (3.50); (b) Equation (3.51); (c) Equation (3.52); (d) Equation (3.53); (e) Equation (3.54) 
the traveling wave solutions for As an application, the traveling wave solutions for Nano-ionic solitons wave's propagation along microtubules in living cells and Nano-ionic currents of MTs, which have been constructed using the $\exp (-\varphi(\xi))$-expansion method. Let us compare our results obtained in the present article with the well-known results obtained by other authors using different methods as follows: Our results of Nano-ionic solitons wave's propagation along microtubules in living cells and Nano-ionic currents of MTs [27]. It can be concluded that this method is reliable and propose a variety of exact solutions NPDEs. The performance of this method is effective and can be applied to many other nonlinear evolution equations.

\section{References}

[1] Maliet, W. (1992) Solitary Wave Solutions of Nonlinear Wave Equation. American Journal of Physics, 60, 650-654. http://dx.doi.org/10.1119/1.17120

[2] Maliet, W. and Hereman, W. (1996) The Tanh Method: Exact Solutions of Nonlinear Evolution and Wave Equations. Physica Scripta, 54, 563-568.

[3] Wazwaz, A.M. (2004) The Tanh Method for Travelling Wave Solutions of Nonlinear Equations. Applied Mathematics and Computation, 154, 714-723. http://dx.doi.org/10.1016/S0096-3003(03)00745-8

[4] EL-Wakil, S.A. and Abdou, M.A. (2007) New Exact Travelling Wave Solutions Using Modified Extended Tanh-Function Method. Chaos, Solitons \& Fractals, 31, 840-852. http://dx.doi.org/10.1016/j.chaos.2005.10.032

[5] Fan, E. (2000) Extended Tanh-Function Method and Its Applications to Nonlinear Equations. Physics Letters A, 277, 212-218. http://dx.doi.org/10.1016/S0375-9601(00)00725-8

[6] Abdelrahman, M.A.E., Zahran, E.H.M. and Khater, M.M.A. (2015) Exact Traveling Wave Solutions for Modified Liouville Equation Arising in Mathematical Physics and Biology. International Journal of Computer Applications (0975 8887), 112.

[7] Wazwaz, A.M. (2005) Exact Solutions to the Double Sinh-Gordon Equation by the Tanh Method and a Variable Separated ODE Method. Computers \& Mathematics with Applications, 50, 1685-1696.

http://dx.doi.org/10.1016/j.camwa.2005.05.010

[8] Wazwaz, A.M. (2004) A Sine-Cosine Method for Handling Nonlinear Wave Equations. Mathematical and Computer Modelling, 40, 499-508. http://dx.doi.org/10.1016/j.mcm.2003.12.010

[9] Yan, C. (1996) A Simple Transformation for Nonlinear Waves. Physics Letters A, 224, 77-84. http://dx.doi.org/10.1016/S0375-9601(96)00770-0

[10] Fan, E. and Zhang, H. (1998) A Note on the Homogeneous Balance Method. Physics Letters A, 246, 403-406. http://dx.doi.org/10.1016/S0375-9601(98)00547-7

[11] Wang, M.L. (1996) Exct Solutions for a Compound KdV-Burgers Equation. Physics Letters A, 213, 279-287. http://dx.doi.org/10.1016/0375-9601(96)00103-X

[12] Zahran E.H.M. and Khater, M.M.A. (2014) The Modified Simple Equation Method and Its Applications for Solving Some Nonlinear Evolutions Equations in Mathematical Physics. Jokull Journal, 64.

[13] Ren, Y.J. and Zhang, H.Q. (2006) A Generalized F-Expansion Method to Find Abundant Families of Jacobi Elliptic Function Solutions of the (2+1)-Dimensional Nizhnik-Novikov-Veselov Equation. Chaos, Solitons \& Fractals, 27, 959-979. http://dx.doi.org/10.1016/j.chaos.2005.04.063

[14] Zhang, J.L., Wang, M.L., Wang, Y.M. and Fang, Z.D. (2006) The Improved F-Expansion Method and Its Applications. Physics Letters A, 350, 103-109. http://dx.doi.org/10.1016/j.physleta.2005.10.099

[15] He, J.H. and Wu, X.H. (2006) Exp-Function Method for Nonlinear Wave Equations. Chaos, Solitons \& Fractals, 30 , 700-708. http://dx.doi.org/10.1016/j.chaos.2006.03.020

[16] Aminikhad, H., Moosaei, H. and Hajipour, M. (2009) Exact Solutions for Nonlinear Partial Differential Equations via Exp-Function Method. Numerical Methods for Partial Differential Equations, 26, 1427-1433.

[17] Zhang, Z.Y. (2008) New Exact Traveling Wave Solutions for the Nonlinear Klein-Gordon Equation. Turkish Journal of Physics, 32, 235-240.

[18] Wang, M.L., Zhang, J.L. and Li, X.Z. (2008) The (G'/G)-Expansion Method and Travelling Wave Solutions of Nonlinear Evolutions Equations in Mathematical Physics. Physics Letters A, 372, 417-423. http://dx.doi.org/10.1016/j.physleta.2007.07.051

[19] Zhang, S., Tong, J.L. and Wang, W. (2008) A Generalized (G'/G)-Expansion Method for the mKdv Equation with Variable Coefficients. Physics Letters A, 372, 2254-2257. http://dx.doi.org/10.1016/j.physleta.2007.11.026

[20] Zayed, E.M.E. and Gepreel, K.A. (2009) The (G'/G)-Expansion Method for Finding Traveling Wave Solutions of Nonlinear Partial Differential Equations in Mathematical Physics. Journal of Mathematical Physics, 50, 013502-013513. 
http://dx.doi.org/10.1063/1.3033750

[21] Zahran, E.H.M. and Khater, M.M.A. (2014) Exact Solutions to Some Nonlinear Evolution Equations by the (G'/G)Expansion Method Equations in Mathematical Physics. Jokull Journal, 64.

[22] Dai, C.Q. and Zhang, J.F. (2006) Jacobian Elliptic Function Method for Nonlinear Differential Difference Equations. Chaos, Solitons \& Fractals, 27, 1042-1049. http://dx.doi.org/10.1016/j.chaos.2005.04.071

[23] Fan, E. and Zhang, J. (2002) Applications of the Jacobi Elliptic Function Method to Special-Type Nonlinear Equations. Physics Letters A, 305, 383-392. http://dx.doi.org/10.1016/S0375-9601(02)01516-5

[24] Liu, S., Fu, Z., Liu, S. and Zhao, Q. (2001) Jacobi Elliptic Function Expansion Method and Periodic Wave Solutions of Nonlinear Wave Equations. Physics Letters A, 289, 69-74. http://dx.doi.org/10.1016/S0375-9601(01)00580-1

[25] Zahran, E.H.M. and Khater, M.M.A. (2014). Exact Traveling Wave Solutions for the System of Shallow Water Wave Equations and Modified Liouville Equation Using Extended Jacobian Elliptic Function Expansion Method. American Journal of Computational Mathematics (AJCM), 4, 455-463.

[26] Rahman, N., Alam, Md.N., Harun-Or-Roshid, Akter, S. and Akbar, M.A. (2014) Application of exp((-Iphi((xxi)) Expansion Method to Find the Exact Solutions of Shorma-Tasso-Olver Equation. African Journal of Mathematics and Computer Science Research, 7, 1-6. http://dx.doi.org/10.5897/AJMCSR2014.0532

[27] Islam, R., Alam, Md.N., A.K.M. Kazi Sazzad Hossain, H.-O.-R. and Akbar, M.A. (2013) Traveling Wave Solutions of Nonlinear Evolution Equations via Exp(-Iphi(|xi))-Expansion Method. Global Journal of Science Frontier Research Mathematics and Decision Sciences, 13, 63-71.

[28] Abdelrahman, M.A.E., Zahran, E.H.M. and Khater, M.M.A. Exact Traveling Wave Solutions for Power Law and Kerr Law Non Linearity Using the exp((-Iphi(|xi)) Expansion Method. GJSFR, 14.

[29] Satarić, M.V., Tuszyński, J.A. and Žakula, R.B. (1993) Kinklike Excitations as an Energy-Transfer Mechanism in Microtubules. Physical Review E, 48, 589-597. http://dx.doi.org/10.1103/PhysRevE.48.589 